\title{
Physiological Fusion of Functional and Structural Data for Cardiac Deformation Recovery
}

\author{
Ken C.L. Wong ${ }^{1,2}$, Linwei Wang ${ }^{1}$, Heye Zhang ${ }^{3}$, and Pengcheng Shi ${ }^{1}$ \\ ${ }^{1}$ Computational Biomedicine Laboratory, Rochester Institute of Technology, Rochester, USA \\ \{linwei.wang, pengcheng.shi\}@rit.edu \\ ${ }^{2}$ ASCLEPIOS Research Project, INRIA Sophia Antipolis, Sophia Antipolis, France \\ kenclwong@sophia.inria.fr \\ ${ }^{3}$ Bioengineering Institute, University of Auckland, Auckland, New Zealand \\ heye. zhang@auckland.ac.nz
}

\begin{abstract}
The advancement in meaningful constraining models has resulted in increasingly useful quantitative information recovered from cardiac images. Nevertheless, single-source data used by most of these algorithms have put certain limits on the clinical completeness and relevance of the analysis results, especially for pathological cases where data fusion of multiple complementary sources is essential. As traditional image fusion strategies are typically performed at pixel level by fusing commensurate information of registered images through various mathematical operators, such approaches are not necessarily based on meaningful biological bases, particularly when the data are dissimilar in physical nature and spatiotemporal quantity. In this work, we present a physiological fusion framework for integrating information from different yet complementary sources. Using a cardiac physiome model as the central link, structural and functional data are naturally fused together for a more complete subject-specific information recovery. Experiments were performed on synthetic and real data to show the benefits and potential clinical applicability of our framework.
\end{abstract}

\section{Introduction}

Cardiac measurements can be categorized as structural and functional. Structural data, such as magnetic resonance images (MRI), noninvasively provide cardiac structures and motions in various spatiotemporal resolutions. Functional data, such as body surface potential maps (BSPM), reveal physiological information of electrical propagation.

To provide physiologically plausible constraints to recover subject-specific information from measurements, cardiac physiome models have been utilized to describe the general macroscopic electromechanical physiology of the heart, which comprise an electrical propagation model (E model) describing electricity propagation within the myocardium, an electromechanical coupling model (EM model) converting the electrical stimulations into contraction stresses, and a biomechanical model (BM model) relating the contraction stresses with the cardiac deformation. In [1], an E model was used to recover volumetric myocardial electrical activities from BSPM. In [2]3], cardiac physiome models were coupled with MRI to estimate ventricular deformation. These applications show that cardiac physiome models are valuable for revealing subject-specific 
cardiac functions. Nevertheless, regardless of the general physiological meaningfulness, these single-source algorithms could not benefit from the complementary information of the other measuring modalities, especially in pathological cases.

To enhance subject-specificity and reliability of the recovery results, measurements from different sources should be fused together to include complementary information. As MRI and BSPM are different manifestations of cardiac functions, fusion of these data aids improving the clinical relevance of the recovered information. In practice, the fusion techniques widely used in medical image analysis are pixel-level fusions, by which the spatial and spectral data of all layers of the registered commensurate images are combined through mathematical operators such as the Boolean or fuzzy logic operators. Nevertheless, the acquisition spaces (myocardium vs. body surface) and physical natures (structure vs. electricity) of MRI and BSPM are incommensurate, the direct use of these operators is physiologically meaningless, if not impossible. In view of the ability to couple subject-specific data from electrical and structural measurements, we propose to adopt a cardiac physiological model as the physiological link for fusing information from these data. Specifically, transmembrane potentials (TMP) recovered from the subject's BSPM through the E model are converted into active stresses through the EM model, which are subsequently related to the myocardial deformation through the cardiac electromechanical dynamics. By coupling this subject-specific a priori deformation with the motion information extracted from the MRI sequence of the same subject under a state-space filtering framework, the cardiac deformation is recovered. In this manner, electrical and structural data are fused together and tightly coupled through the cardiac physiome model. Experiments were performed on synthetic and real data to show the benefits and potential clinical applicability.

\section{Cardiac Physiome Model}

The cardiac physiome model acts as the physiological link between the electrical and structural information. Only the E model and the cardiac electromechanical dynamics are presented, as they directly associate with the data fusion.

\subsection{Electric Wave Propagation Model}

To model the TMP propagation of the myocardium, the E model described by a twovariable diffusion-reaction system is used, which matrix representation is given as [1]:

$$
\frac{\partial \mathbf{U}_{\mathrm{E}}}{\partial t}=-\mathbf{M}_{\mathrm{E}}^{-1} \mathbf{K}_{\mathrm{E}} \mathbf{U}_{\mathrm{E}}+f_{1}\left(\mathbf{U}_{\mathrm{E}}, \mathbf{V}\right) ; \quad \frac{\partial \mathbf{V}}{\partial t}=f_{2}\left(\mathbf{U}_{\mathrm{E}}, \mathbf{V}\right)
$$

where $\mathbf{U}_{\mathrm{E}}$ and $\mathbf{V}$ are vectors of nodal TMP and repolarization variables respectively. Matrices $\mathbf{M}_{\mathrm{E}}$ and $\mathbf{K}_{\mathrm{E}}$ account for the intercellular electrical propagation. $f_{1}$ and $f_{2}$ are general descriptors of cellular TMP dynamics.

\subsection{Cardiac Electromechanical Dynamics}

The TMP can be converted into active forces through the EM model, which are further related to the cardiac deformation through the cardiac electromechanical dynamics. 


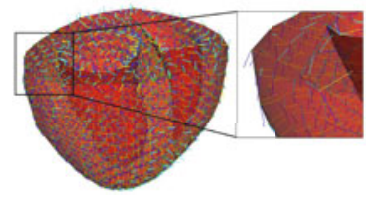

(a)

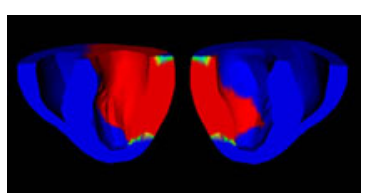

(b)

Fig. 1. Synthetic data. (a) Heart represented as nodes bounded by surfaces, with tissue structures indicated by local coordinate systems (fiber, sheet, sheet normal: blue, yellow, cyan). (b) Infarcted regions shown in red color.

The displacement-based total-Lagrangian system dynamics is utilized, which is given as [3]:

$$
\mathbf{M}_{\mathrm{BM}} \ddot{\mathbf{U}}_{\mathrm{BM}}+\mathbf{C} \dot{\mathbf{U}}_{\mathrm{BM}}+\mathbf{K}_{\mathrm{BM}} \Delta \mathbf{U}_{\mathrm{BM}}=\mathbf{F}_{c}-\mathbf{F}_{i}
$$

where $\mathbf{M}_{\mathrm{BM}}, \mathbf{C}$, and $\mathbf{K}_{\mathrm{BM}}$ are the mass, damping, and stiffness matrices respectively. The hyperelastic and orthotropic material properties of the BM model are embedded in $\mathbf{K}_{\mathrm{BM}} . \mathbf{F}_{c}$ is the force vector containing the active forces from the $\mathrm{E}$ and EM models. $\mathbf{F}_{i}$ is the force vector related to internal stresses. $\ddot{\mathbf{U}}_{\mathrm{BM}}, \dot{\mathbf{U}}_{\mathrm{BM}}$ and $\Delta \mathbf{U}_{\mathrm{BM}}$ are the respective acceleration, velocity and incremental displacement vectors respectively.

\section{Physiological Data Fusion}

To fuse structural and functional image information, subject-specific volumetric TMP are first recovered from BSPM through the E model, which become the force inputs to the cardiac electromechanical dynamics through the EM model. This BSPM-embedded cardiac electromechanical dynamics is then used to recover cardiac deformation from MRI with fused information from both functional and structural images.

\subsection{Functional Data and Volumetric Electrophysiological Recovery}

To relate BSPM to the cardiac physiome model, the framework in [1] is used to image volumetric TMP from BSPM. Noninvasive observations of TMP are provided by potential maps on the body surface, with their relation follows the quasi-static electromagnetism on the personalized heart-torso model obtained from subject's MRI. The resulted linear relation between volumetric TMP and BSPM is given as:

$$
\mathbf{y}_{\mathrm{E}}=\mathbf{H}_{\mathrm{E}} \mathbf{U}_{\mathrm{E}}
$$

where $\mathbf{y}_{\mathrm{E}}$ consists of the body surface potentials (BSP). This model combines the advantage of FEM-based and BEM-based approaches to TMP-to-BSP modeling.

As $\mathbf{U}_{\mathrm{E}}$ is of much higher dimension than $\mathbf{y}_{\mathrm{E}}$, the $\mathrm{E}$ model (1) is necessary to provide the physiological constraint for obtaining a unique $\mathrm{U}_{\mathrm{E}}$ from $\mathrm{y}_{\mathrm{E}}$. To account for the uncertainties of the model and the measurements, (1) and (3) are reformulated into state-space representations to utilize optimal estimation [4]. By letting the state vector 

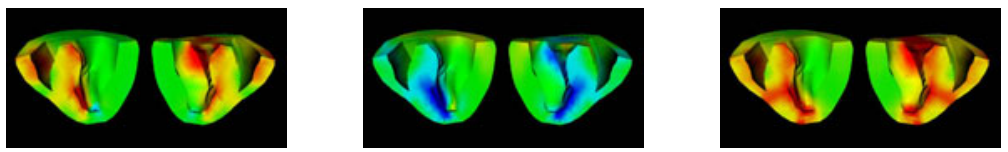

(a) Simulated ground truth.
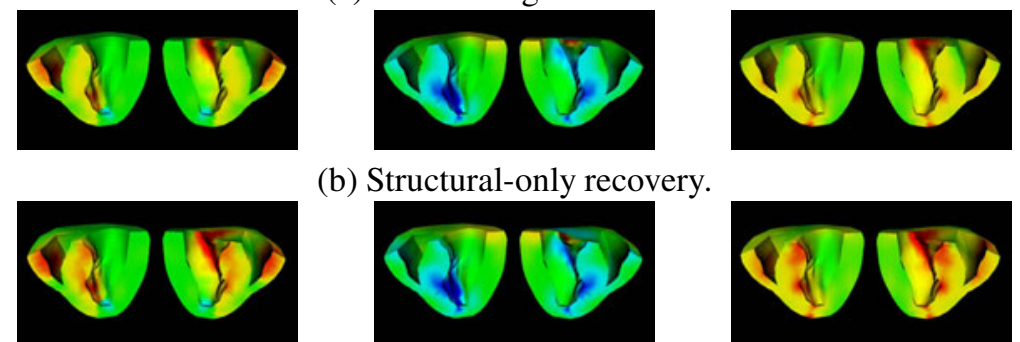

(b) Structural-only recovery.
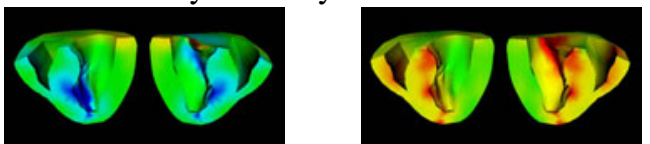

(c) Fusion-based recovery.
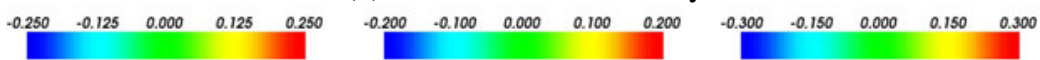

Fig. 2. Synthetic data. Strain maps at the end of systole $(250 \mathrm{~ms})$, with red, blue, and green colors representing extension, contraction, and no deformation respectively. Left to right: radial strain, circumferential strain, and first principal strain.

$\mathbf{x}_{\mathrm{E}}^{\mathrm{T}}=\left[\mathbf{U}_{\mathrm{E}}^{\mathrm{T}}, \mathbf{V}^{\mathrm{T}}\right]$, and the model uncertainties and measurement errors as $\boldsymbol{\omega}_{\mathrm{E}}$ and $\boldsymbol{\nu}_{\mathrm{E}}$ respectively, the state-space system is given as:

$$
\begin{aligned}
& \mathbf{x}_{\mathrm{E}}(k)=f_{\mathrm{E}}\left(\mathbf{x}_{\mathrm{E}}(k-1)\right)+\boldsymbol{\omega}_{\mathrm{E}}(k-1) \\
& \mathbf{y}_{\mathrm{E}}(k)=\tilde{\mathbf{H}}_{\mathrm{E}} \mathbf{x}_{\mathrm{E}}(k)+\boldsymbol{\nu}_{\mathrm{E}}(k)
\end{aligned}
$$

with $f_{\mathrm{E}}$ rearranged from (1) and discretized in time, and $\tilde{\mathbf{H}}_{\mathrm{E}}=\left[\mathbf{H}_{\mathrm{E}}, \mathbf{0}\right]$. With (45), given a subject's BSPM sequence, volumetric TMP can be estimated through the statespace filtering in Section 3.3, which can then be transformed into contraction forces through the EM model, and embedded to the cardiac electromechanical dynamics.

\subsection{Structural Data and Electrophysiology-Guided Deformation Recovery}

To relate the structural images with the cardiac deformation, the relation between the nodal displacements $\mathbf{U}_{\mathrm{BM}}$ and the image-extracted motions $\mathbf{y}_{\mathrm{BM}}$ is given as:

$$
\mathbf{y}_{\mathrm{BM}}=\mathbf{H}_{\mathrm{BM}} \mathbf{U}_{\mathrm{BM}}
$$

where $\mathbf{H}_{\mathrm{BM}}$ is the measurement matrix with interpolation functions relating $\mathbf{U}_{\mathrm{BM}}$ to $\mathbf{y}_{\mathrm{BM}}$. For a unique solution, $\mathbf{U}_{\mathrm{BM}}$ is related to the BSPM-embedded cardiac dynamics through (2), and this establishes the relation between the structural images and the BSPM. As (2) is already discretized in time, by letting the state vector $\mathbf{x}_{\mathrm{BM}}$ as $\mathbf{U}_{\mathrm{BM}}$ and the model uncertainties as $\boldsymbol{\omega}_{\mathrm{BM}}$, the state-update equation is given as:

$$
\begin{aligned}
\mathbf{U}_{\mathrm{BM}}(k) & =\mathbf{U}_{\mathrm{BM}}(k-1)+\Delta \mathbf{U}_{\mathrm{BM}}+\boldsymbol{\omega}_{\mathrm{BM}}(k-1) \\
\Rightarrow \mathbf{x}_{\mathrm{BM}}(k) & =f_{\mathrm{BM}}\left(\mathbf{x}_{\mathrm{BM}}(k-1)\right)+\boldsymbol{\omega}_{\mathrm{BM}}(k-1)
\end{aligned}
$$



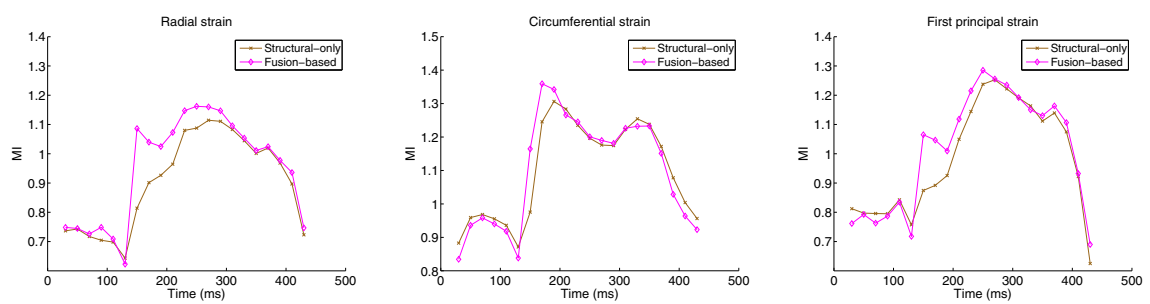

Fig. 3. Synthetic data. Mutual information between strain patterns of the ground truth and the recoveries, for the whole cardiac cycle.

with $\Delta \mathbf{U}_{\mathrm{BM}}$ obtained by solving (2), thus $f_{\mathrm{BM}}$ is nonlinear with respect to $\mathrm{x}_{\mathrm{BM}}$. The corresponding stochastic measurement equation is obtained from (6) as:

$$
\mathbf{y}_{\mathrm{BM}}(k)=\mathbf{H}_{\mathrm{BM}} \mathbf{x}_{\mathrm{BM}}(k)+\boldsymbol{\nu}_{\mathrm{BM}}(k)
$$

where $\boldsymbol{\nu}_{\mathrm{BM}}$ accounts for the measurement errors. With this system, the state-space filtering framework in Section 3.3 can be used to estimate subject-specific cardiac deformation with physiologically fused information from both BSPM and structural images.

\subsection{Nonlinear State-Space Filtering for Model-Measurement Coupling}

System (45) and system (718) are in the same form:

$$
\begin{aligned}
& \mathbf{x}(k)=f(\mathbf{x}(k-1))+\boldsymbol{\omega}(k-1) \\
& \mathbf{y}(k)=\mathbf{H} \mathbf{x}(k)+\boldsymbol{\nu}(k)
\end{aligned}
$$

In consequence, the same filtering framework can be applied. To preserve model nonlinearity and maintain computational feasibility, we utilize the unscented Kalman filter (UKF) which comprises the advantages of Monte Carlo methods and Kalman filter updates [4]. In UKF, the prediction step is done by the unscented transformation, which calculates the statistics of a random variable undergoing a nonlinear transformation. A set of minimal sample points called sigma points is carefully chosen from the state statistical distribution, which completely captures the true mean and covariance. Each sigma point is projected with intact nonlinearity through (9), and all projected points are combined together for the projected mean and covariance. Afterwards, as (10) is linear, the correction procedures of Kalman filtering can be applied to obtain the estimation.

\section{Experiments}

\subsection{Synthetic Data}

The heart architecture from the University of Auckland was used to provide the anatomical cardiac geometry and tissue structure for the experiments [5] (Fig. 1]a)). To show that our framework can aid verifying possible diseased areas, part of the heart was assumed to be infarcted (Fig.1 b)), where the mechanical stiffness was three times as that 

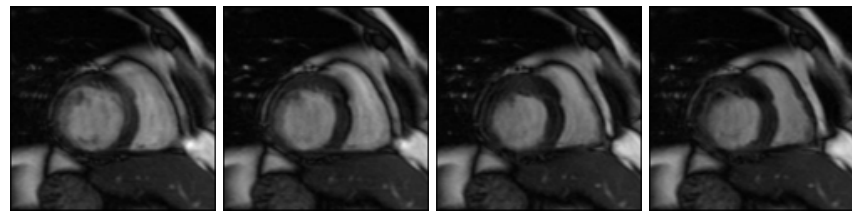

Fig. 4. Human data, case 2. MRI sequence during systole (frame \#1, \#3, \#5, \#7).

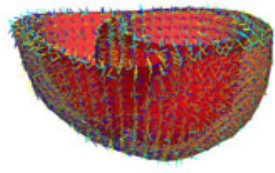

(a)

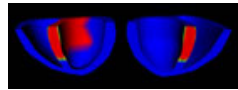

(b)

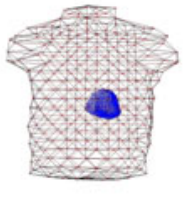

(c)

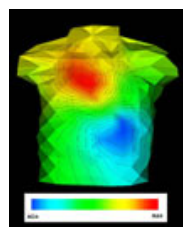

(d)

Fig. 5. Human data, case 2. (a) Cardiac geometry segmented from MRI, with mapped tissue structures. (b) Infarcted segments $(3,4,9,10)$ shown in red color. (c) Patient's heart-torso representation. (d) Exemplary input BSPM of ventricular activation.

of the normal tissue, and the cardiac electricity could not propagate through. A cardiac cycle of $450 \mathrm{~ms}$ was simulated through the cardiac physiome model as the ground truth. The nodal displacements on the heart boundaries were extracted, and added with noises of $5 \mathrm{~dB}$ SNR as the noisy structural measurement inputs of the experiments.

Two frameworks were tested. The first framework has no active force $\mathbf{F}_{c}$, so only structural measurements were used (structural-only recovery). The second framework is our fusion-based recovery with both subject-specific (abnormal) TMP and structural measurements. In the recovery process, the infarcted regions were unknown.

Fig. 2] shows the strain maps at the end of systole. For both frameworks, the recovered strains show almost no deformation in the infarcted regions. Comparing between the frameworks, the radial and first principal strains recovered by the fusion-based framework are closer to the ground truth than those recovered by the structural-only framework, while the circumferential strains of both frameworks are similar. To quantify the similarities between strain patterns of the ground truth and the recoveries, mutual information (MI) comparison is utilized. Fig. 3 shows that similar to the observations of the strain maps, the recovered strains using the fusion-based framework are closer to the ground truth in general, especially during systole (120 ms to $250 \mathrm{~ms})$.

\subsection{Human Data}

To show the potential clinical applicability of our framework, experiments were done on human data sets comprising both BSPM and MRI, which are case 1 and case 2 available at [6], from two patients with acute myocardial infarction. Case 1 contains a human short-axis MRI sequence of 19 frames $(52.5 \mathrm{~ms} /$ frame), with 12 slices/frame. Case 2 contains a human short-axis MRI sequence of 16 frames $(50 \mathrm{~ms} / \mathrm{frame})$, with 


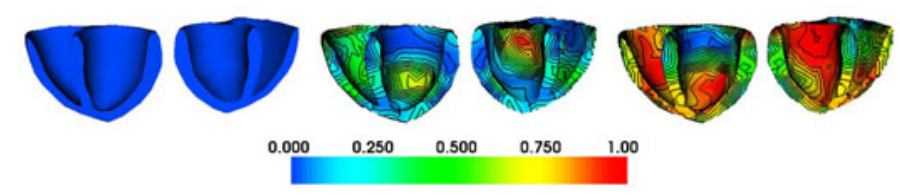

Fig. 6. Human data, case 2. Normalized volumetric myocardial TMP recovered from patient's BSPM, shown with isochrones. Left to right: $0 \mathrm{~ms}, 5 \mathrm{~ms}$, and $12 \mathrm{~ms}$.
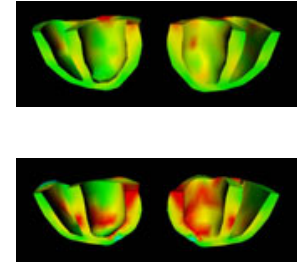

(a) Structural-only recovery.

(b) Fusion-based recovery.

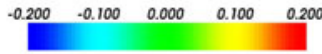

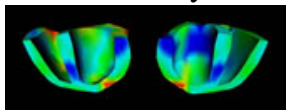
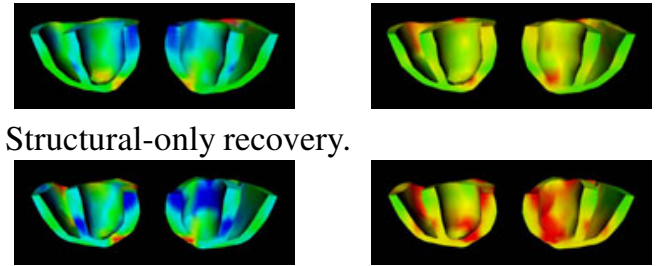

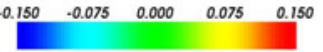

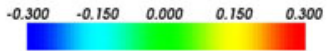

Fig. 7. Human data, case 2. Strain maps at the end of systole (300 ms). Left to right: radial strain, circumferential strain, and first principal strain.

13 slices/frame. Both sequences have $8 \mathrm{~mm}$ inter-slice spacing, and in-plane resolution $1.32 \mathrm{~mm} /$ pixel (Fig. 4). Each case has the patient's BSPM recorded from 123 electrodes and interpolated to 370 nodes on the torso surface. Segmentations were performed to obtain the initial heart geometries, with the fibrous-sheet structures mapped from the Auckland heart architecture using nonrigid registration (Fig.5 (a)). The expert-identified infarcted segments of case 1 are $1,2,3,8,9,13,14,15$, and of case 2 are 3, 4, 9, 10, with respect to the myocardial nomenclature in [7] (Fig.5]b)). Fig. 5(c) illustrates the combined heart-torso model of the patient, where the torso was obtained through segmentation of the patient's torso MRI. Fig. 5(d) exemplifies an input BSPM.

In the experiments, the volumetric TMP were first recovered from the BSPM using the system described in Section 3.1. The recovered TMP show limited propagation in the infarcted regions, which is different from the normal regions (Fig. 6).

Motions of the heart boundaries were extracted from MRI using free-form image registration between consecutive image frames [8]. Experiments were performed using the structural-only framework and the fusion-based framework, on both case 1 and case 2. Fig. 7 shows that although both frameworks show relatively small deformation in the infarcted regions, the fusion-based framework has a better contrast between the normal and the infarcted regions, and the shapes of the regions with negligible deformation are more consistent with the shapes of the infarcted regions.

For clinical applications, the capabilities of frameworks to aid locating diseased regions are of great interest, thus the frameworks are compared in this aspect. As there is a strong inverse relationship between the first principal strain and the extent of myocardial infarction [9], we sorted the segments at different levels according to the mean 
Table 1. Human data. Segment numbers at different levels sorted according to the mean of the first principal strains of each segment at the end of systole, in ascending order. The numbers of the infarcted segments are highlighted in red color.

\begin{tabular}{|c|c|c|c|c|c|c|c|}
\hline$\overline{\overline{\text { Case } 1}}$ & $\overline{\text { Basal }}$ & $\overline{\mathrm{Mid}}$ & $\overline{\overline{\text { Apical }}}$ & $\overline{\text { Case } 2}$ & $\overline{\text { Basal }}$ & $\overline{\mathrm{Mid}}$ & $\overline{\text { Apical }}$ \\
\hline Structural-only & 342516 & 1098871112 & \begin{tabular}{|lllll}
14 & 15 & 13 & 16
\end{tabular} & Structural-only & $\begin{array}{llllll}3 & 5 & 2 & 1 & 4 & 6\end{array}$ & $\begin{array}{llllll}10 & 9 & 11 & 12 & 8 & 7\end{array}$ & $\begin{array}{lllll}14 & 15 & 16 & 13\end{array}$ \\
\hline \begin{tabular}{|l} 
Fusion-based \\
\end{tabular} & 324156 & 1098871112 & $\begin{array}{llll}14 & 13 & 15 & 16\end{array}$ & Fusion-based & 341256 & $\begin{array}{llllll}10 & 9 & 11 & 7 & 8 & 12\end{array}$ & $14 \quad 15 \quad 16 \quad 13$ \\
\hline
\end{tabular}

Table 2. Human data. Optimal sensitivities (SEN) and specificities (SPEC) of the frameworks for identifying infarcted segments.

\begin{tabular}{|c|c|c|c|}
\hline \hline \multirow{2}{*}{ Case 1 } & Basal & Mid & Apical \\
\cline { 2 - 4 } & SEN/SPEC & SEN / SPEC & SEN / SPEC \\
\hline Structural-only & $67 \% / 67 \%$ & $100 \% / 75 \%$ & $100 \% / 100 \%$ \\
\hline Fusion-based & $100 \% / 67 \%$ & $100 \% / 75 \%$ & $100 \% / 100 \%$ \\
\hline
\end{tabular}

\begin{tabular}{|c|c|c|}
\hline \hline \multirow{2}{*}{ Case 2 } & Basal & Mid \\
\cline { 2 - 3 } & SEN/SPEC & SEN / SPEC \\
\hline Structural-only & $50 \% / 100 \%$ & $100 \% / 100 \%$ \\
\hline Fusion-based & $100 \% / 100 \%$ & $100 \% / 100 \%$ \\
\hline
\end{tabular}

of the first principal strains of each segment at the end of systole, in ascending order (Table 1). From Table 1 we can calculate the various sensitivities and specificities with different cutoff segments, where sensitivities are the ratios between the correctly identified positives (infarcted segments) and the actual positives, and the specificities are the ratios between the correctly identified negatives (normal segments) and the actual negatives. Table 2 shows that for both cases, the fusion-based framework achieves the same or higher optimal sensitivities compared with the structural-only framework.

\section{References}

1. Wang, L., Zhang, H., Wong, K.C.L., Liu, H., Shi, P.: Noninvasive volumetric imaging of cardiac electrophysiology. In: IEEE CVPR, pp. 2176-2183 (2009)

2. Sermesant, M., Delingette, H., Ayache, N.: An electromechanical model of the heart for image analysis and simulation. IEEE Transactions on Medical Imaging 25(5), 612-625 (2006)

3. Wong, K.C.L., Wang, L., Zhang, H., Shi, P.: Nonlinear cardiac deformation recovery from medical images. In: IEEE ICIP, pp. 2513-2516 (2009)

4. Simon, D.: Optimal State Estimation: Kalman, $\mathrm{H}_{\infty}$, and Nonlinear Approaches. John Wiley \& Sons, Inc., Chichester (2006)

5. Nash, M.: Mechanics and Material Properties of the Heart using an Anatomically Accurate Mathematical Model. PhD thesis, The University of Auckland (1998)

6. PhysioNet/Computers in Cardiology challenge 2007: electrocardiographic imaging of myocardial infarction (2007), http: / / www . physionet.org/challenge/2007/

7. Cerqueira, M.D., Weissman, N.J., Dilsizian, V., Jacobs, A.K., Kaul, S., Laskey, W.K., Pennell, D.J., Rumberger, J.A., Ryan, T., Verani, M.S.: Standardized myocardial segmentation and nomenclature for tomographic imaging of the heart: a statement for healthcare professionals from the cardiac imaging committee of the council on clinical cardiology of the American Heart Association. Circulation 105, 539-542 (2002)

8. Perperidis, D., Mohiaddin, R.H., Rueckert, D.: Spatio-temporal free-form registration of cardiac MR image sequences. Medical Image Analysis 9(5), 441-456 (2005)

9. Tim Marcus, J., Götte, M.J.W., van Rossum, A.C., Kuijer, J.P.A., Heethaar, R.M., Axel, L., Visser, C.A.: Myocardial function in infarcted and remote regions early after infarction in man: assessment by magnetic resonance tagging and strain analysis. Magnetic Resonance in Medicine 38(5), 803-810 (1997) 